country ${ }^{4}$. The fact that there is widespread infection in wild rabbits but only a few authentic infections of tame rabbits is also evidence against participation of a winged vector. On the other hand, rabbit fleas (Spilopsyllus cuniculi), which do not occur in Australia, may well be acting as a vector here. I have found, as have others, that among rabbits kept free from ectoparasites, cage-to-cage infection in the laboratory has not occurred. Fleass are abundant on wild rabbits and with warmer weather congregate more on the head where myxomatous lesions chiefly occur. Though specific parasites of rabbits, they are occasionally carried on birds and presumably could in this way help in wider spread of infection ${ }^{5}$.

\section{Changes in Rabbit-virus Relationship}

A relationship in which a parasite kills more than 99 per cent of its hosts is not likely to be stable. In associations of long standing, host and parasite become adapted to living together, as is the case with myxoma virus and the South American tapeti. An adjustment in this sense is sure to take place elsewhere. Development of inherited resistance is likely to take a long time in rabbits-even they are slowly breeding beings in comparison with a virus. Thero is so far little evidence that offspring of recovered rabbits are resistant. On the other hand, there have been found in Australia virus strains of lowered virulence, taking longer to kill and killing only 90 per cent instead of more than 99 per cent. Rabbits living longer remain infectious longer and the virus can therefore spread more readily, so that one with a tendency to kill more slowly has an evolutionary advantage ${ }^{6}$. So far there is little or no evidence of emergence of an attenuated virus in Britain, or, so far as one knows, in Europe. Indeed, the apparently more stable European strain is being used for re-introductions of virus into Australia.

\section{Human Intervention and Myxomatosis}

In Australia, even whore the kill of rabbits is very high, the disease passes on, and surviving rabbits begin to breed. The new generation of rabbits will not be immune and to keep them down and, as the Australians say, to "back up the virus", other destructive measures are being used and, when needful, virus is re-introduced. Thus, myxomatosis is greatly helping Australian economy; but to take full advantage of it, scientific "backing-up" is being used on a very large scale.

In the British Isles, myxomatosis has now reached nearly every county of England and Wales and a number in Scotland and Ireland. Its incidence, however, is very patchy. In most counties one can walk for miles and see plenty of healthy rabbits and not a single diseased ono. The total destruction of rabbits must be still trivial. 'The poor summer of 1954 may have militated against spread by 'flying pins', and a warm September might yet alter the picture. It seems likely, however, that my xomatosis, except perhaps in south-east England, is only here and there. Things might remain like this into the indefinite future, with the disease spreading naturally or otherwise from farm to farm, parish to parish and back to its starting point as new susceptible rabbits grow up. There might well be no striking effect on rabbit numbers, except indirectly as the existence of the disease affected the trapping industry and the taste of the people for rabbit-meat; these factors might leave us in many areas with more rabbits rather than less. Further, we should still have the disease with us, with local epidemics killing the rabbits in hundreds in its highly unpleasant fashion. If such should indeed be the outcome, we should have all the disadvantages of myxomatosis with no compensating economic gain. It might then be cogently argued that we should follow the Australian example, introduce the disease simultaneously into areas of convenient size and "back it up" intensively. "The rabbit population might thus be drastically reduced with a resultant gain not only to our economy but also to our very natural wish to inflict the least possible suffering on the rabbits. Maximum suffering over a long period would be caused if the disease kept going spontaneously round the country like a travelling circus. Scientists did not introduce the disease into Britain, and no official or unofficial action of man can possibly stop it. What the disease will a.ccomplish 'on its own' we cannot yet foresee.

${ }^{1}$ A ragão, H. de B., Memorias do Ist. Oswaldo Cruz, 20, 225 (192S). ${ }^{2}$ Report of Advisory Committee on Myxomatosis (II.M. Stationery Office, 1954).

Rateliffe, F. N., Myers, K., Fennessy, I3. V., and Calaby, J. H., Nature, 170,7 (1952).

1 Muirhead Thomsun, R. C. (personal communication).

${ }^{5}$ Rothschild, M. (personal communication).

- Fenner, F., Nature, 172, 228 (1955).

\section{NUCLEAR AND MESON PHYSICS}

\section{INTERNATIONAL CONFERENCE IN GLASGOW}

$T$

HE International Union of Pure and Applied Physics and the United Nations Educational, Scientific and Cultural Organization sponsored a conference on nuclear and meson physics which was held in the Department of Natural Philosophy, University of Glasgow, during July 13-17, under the general chairmanship of Prof. P. 1. Dee. A wide range of topics was covered, and more than one hundred papers were presented. In these circumstances it is impossible, in a short review, to do justice to, or even mention, all the contributions.

The opening session was on nuclear forces and nucleon scattering. The present state of knowledge in this field was surveyed by Prof. H. S. W. Massey, who stressed the need for more work on the very light nuclei, in addition to that on proton-proton and proton-neutron scattering, to try to gain some direct evidence on the presence or absence of many-body forces. 'The recent work on nucleon-nucleon scattering at Harvard and Brookhaven was described by Prof. N. F. Ramsey; the extension of proton-proton scattering experiments to high energy has shown that the apparent constancy of the total cross-section at high energies is due to a broad flat minimum in the range $200-400 \mathrm{MeV}$.

In the angular distribution of proton-deuteron scattering at $20.6 \mathrm{MeV}$, described by Dr. D. 0 . Caldwell, a minimum at small angles has been found, due to the interference between the Coulomb and nuclear scattering. This interference should enable data to be fitted with a unique set of phase shifts and decide between the two sets which both fit the earlier data. Work was also reported from several laboratories on the scattering of polarized nucleon beams.

An account of the present status of the meson theory of nuclear forces by M. M. Levy and R. E. 
Marshak was presented by Prof. Marshak, who emphasized the different approximations used in current treatments. He made it clear that, although the present theory includes many of the observed features of nuclear forces, further developments towards a fully non-static approximation are required.

The sessions on nuclear data and nuclear models was opened with review papers: by Prof. J. A. Wheeler on nuclear models, and by Prof. S. Devons on the experimental determination of energy-levels. Prof. Wheeler dealt with tho work currently in progress on different aspects of the problem and, in particular, described the work of $\mathbf{K}$. A. Brueckner. This considers a test nucleon passing through an infinite nuclear medium. The wave describing the nucleon is scattered by each of the moving centres in the medium, the forward-scattering amplitudes adding coherently to the primary wave. No elementary law of interaction enters the calculationsonly the phase shifts which describe the nucleonnucleon scattering up to $100 \mathrm{MeV}$. The present experimental information; however, is not sufficiently complete to exploit this approach.

Prof. Devons emphasized the great theoretical and experimental advances of the past few years, and pointed out that in some cases theory has now out. stripped experiment. He instanced the small differences in the Coulomb energy for corresponding levels of a $T$-multiplet (which have considerable interest, but are not always known with great accuracy) and the low-lying rotational levels of some heavy nuclei which need to be determined with the highest precision. Of great interest was his description of the measurement at the Imperial College of Science and Technology, London, of the reduction of the Doppler shift as a recoil particle passes through slowing-down materials. This method promises to fill the gap in the lifetime measurements of radiative transitions. Of the numerous interesting experimental papers read at this session, that of Dr. W. W. Buechner describing the work at the Massachusetts Institute of Technology, using a magnetic spectrograph as a particle analyser, most emphasized the great accuracy attainable with present-day techniques. A theoretical paper of particular significance was that of $\mathrm{J}$. P. Elliot and B. H. Flowers, who derived the even parity, low-lying levels of mass-19 nuclei. These are assumed to arise from configurations of the form $\Sigma d^{n-x_{\mathcal{S}} x}(x=1,2$ or 3$)$. By using essentially only $x$

one free parameter, the positions and spins of these levels are predicted and show good agreement with the somewhat sparse experimental data. Prof. V. F. Weisskopf closed these sessions with a stimulating and provocative summary of the present position. The problems he discussed included that of the effective mass of a nucleon inside the nucleus, and the possibility of the internucleon forces in nuclei being different from those between free nucleons.

Another aspect of low-energy nuclear physics was discussed at the session on photo-disintegration. The results of Dr. H. W. Koch on X-ray scattering were interpreted by Dr. U. Fano as showing that the giant resonance does not possess a detailed structure. A tentative theory of the giant resonances was put forward by Dr. D. H. Wilkinson. This explains the resonances in terms of single-particle transitions for the absorption process, and the anomalously large yield of high-energy particles and their angular distribution are predicted in terms of the same absorption process.
The field of beta- and gamma-ray transitions was surveyed by Dr. K. Siegbahn, who described the recent developments which enable extremely accurate measurements to be made. A most stimulating talk on the interaction in $\beta$-decay was given by Dr. C. S. Wu. The Fermi and Gamow-Teller components have approximately the same magnitude, and recoil experiments on helium-6 show that the latter is a. tensor interaction. Preliminary results on neon-19 recoils suggest that the Fermi interaction is scalar, as favoured by other evidence. The more recent calculations which nullify the necessity for the pseudoscalar component have been confirmed in their conclusion by the direct measurement of the spin of radium $\mathrm{E}$ by $\mathrm{K}$. Smith, who has obtained a value of 1 .

The conference was divided into two halves: during the first two days nuclear phenomena not involving $\pi$-mesons directly were discussed, and the last two were mainly devoted to mesonic ylargely $\pi$-mesonic) physics. The intervening day, July 15, was spent in a chartered steamer sailing among some of the lochs and islands of the Firth of Clyde; this not only allowed a view of some beautiful scenery, but also gave the delegates an opportunity for discussion, particularly valuable by reason of the rather tight programme.

Prof. H. A. Bethe opened the morning session on July 16 with a general survey of pion-nucleon interactions. He discussed the cross-sections for pionproton scattering up to about $200 \mathrm{MeV}$. which are interpretable in terms of phase shifts for angular momentum states $P_{3 / 2}, P_{1 / 2}$ or $S_{1 / 2}$ and states of total isotopic spin $T=3 / 2$ or $T=1 / 2$. Of the three possible analyses into phase shifts, only one gives a resonance in the $P_{3} T=3 / 2$ state, but physical reasons are given for this being the most plausible analysis. These results, including the low-energy $P$-wave scattering, are interpreted in terms of the pseudoscalar coupling theory of mesons to nucleons, using Tamm-Dancoff approximate methods. The more difficult interpretation of the photo-production cross-sections was also discussed. In this session, papers on experimental researches on pion-nucleon scattering and pion production up to energies of I.5 BeV. were presented by Dr. R. H. Hildebrand (Chicago), Dr. J. Ashkin (Carnegie Institute of Technology), Dr. J. B. Platt (Rochester, N.Y.) and Dr. A. C. Helmholtz (Berkeley).

In a session on detailed meson theory, Prof. F. J. Dyson gave an account of the surmounting of difficulties, in the Tamm-Dancoff method, at Cornell. Other papers included two on the origin of spin orbit coupling in nuclei, while Dr. M. Cini reported the application of the variational method of Cini and Fubini to pion-nucleon scattering. This latter method gives a correct fit of the $P$-wave, though not the $S$-wave, phase shifts to the 'Glicksman set' of phase shifts, and seems capable of refinement by, for example, the introduction of a further variational parameter.

The afternoon of July 16 consisted of four simultaneous meetings of special interest. These were on $\beta$ and $\gamma$ transitions and mesic X-rays, detailed theory of nuclear models, field theory and photodisintegration (mentioned earlier).

Dr. J. Ashkin reported on measurements of the intensities and energies of $X$-rays from $\pi$-mesic atoms. Energy measurements of $\mathrm{X}$-rays from transitions between outer shells, where the $\pi^{-}$-meson nucleon interaction does not appear, gives a close determina. 
tion of the $\pi^{-}$mass, while measurements of line shifts in inner shells where the nuclear interaction operates can be related to phase shifts in the meson-nucleon scattering. Dr. J. B. Platt reported work on the relative intensities of X-ray lines in order to find the attenuation resulting from nuclear capture from inner shells.

The field theory meeting was opened with a talk by Prof. W. Heisenberg on "Quantization of Nonlinear Wave Equations" ; it is proposed to introduce just one wave function for all matter, obeying a nonlinear field equation. The equation

$$
\gamma_{\nu} \frac{\partial \psi}{\partial x_{\nu}}+l^{2} \psi(\psi+\psi)=0
$$

is taken as an example, and study of the likely commutation rules leads to the conclusion that the laws of quantum mechanics would be modified in a certain region of Hilbert space.

The conference ended on the afternoon of July 17 with a full meeting of all delegates, at which papers on heavy mesons and hyperons were presented. Dr. C. C. Butler opened the session with an excellent review of our present knowledge of heavy mesons and hyperons, and the conference closed on a high note with a paper by Drs. A. Pais and M. Gell-Mann on some theoretical speculations concerning the heavy particles, which included an interpretation of $\Lambda^{0}$ particles as particles of high intrinsic spin. The full proceedings of the conference will be published by the Pergamon Press.

E. H. Bellamy

R. G. Moorhouse

\section{SCIENCE, ANIMALS AND HUMANITY*}

BY W. LANE-PETTER

Laboratory Animals Bureau, Medical Research Council

Laboratories, London, N.W.3

$I^{\mathrm{F}}$

I were a psychologist I might understand, and even be able to explain, why animals, and in particular certain animals, evoke an emotional response in human beings, so strong in some that it is beyond the reach of reason. We all know of the cat lovers who carry their devotion outside the limits of economy or hygiene; of the dogs who lay undisputed claim to the best armchair or the master's place on the hearth-rug ; of the horses, canaries and other animals who receive more consideration from their owners than persons more nearly related zoologically and therefore having, one would think, better claims to affection. On the other hand, by taking some animals into domestication, or keeping others in captivity, man accepts an obligation to look after them, for they are entirely dependent on man for the necessities of their being. It makes it easier for us to do our duty towards our animals if we are fond of them.

Emotion, then, is bound to creep into any diseussion of relations between animals and man, and it is a deep and often disturbing emotion. To ignore it is unrealistic, and to be ashamed of it unnecessary ; but it should not be allowed to cloud our reason, any more than any other emotion. Such undisciplined feeling is often associated with a morbid preoccupation with brutality-a revealing word-in books and

* Substance of a paper read on September 6. 1954, before Section D (Zoology) of the British Association meeting at Oxford. articles, on the cinema screen, in conversation and perhaps in real life, and the minutiæ of violence excite a thrill of disgust, guilty pleasure or any other emotion; but above all a thrill. There can be few normal people who are totally unaffected by the sight or image of injury, be it accidental, criminal or surgical; but in most of us that basic attractionrepulsion is clothed in reason or rationalization. So in a discussion of animals let us try to recognize the shadow of the brute within our souls and keep him chained, and let us be especially wary lest any moralizing we may indulge in be the brute tugging at his leash.

There exists a great gulf between those whose primary interest is in the welfare of animals, and the scientists who use animals in their work. It is a gulf of misunderstanding so wide that the leaders in each field are often not on speaking terms with one another, at least in public. "Callous torturer", says the humanitarian, and the scientist replies "sentimental crank". Yet the first may be a reasonable, intelligent and responsible person, and the second as deeply moved by cruelty as any of his fellows, for whom the obligation of humanity is so obvious that he does not bother to explain it. But of course he should explain what he is about, and the layman who criticizes him should listen to the explanations.

What Dr. J. Bronowski says of the nuclear scientist ${ }^{1}$ has a more general application. The scientist, he says, ". . . has one special responsibility . . of enlightenment in scientific matters .. . the responsibility of knowledge, not of power . . . to illuminate for all men the choice which his work offers them. . . Outside his laboratory, his task is to educate us in what goes on inside it, and to give it a meaning for us. In a world in which statesmen as much as voters are ignorant of the simplest implications in science, this is a formidable responsibility ... [the scientist] has no other choice to-day but patiently to become a teacher, in a world in which distrust and prejudice are free. . . There is no alternative to an informed public opinion: and that can exist only where scientists speak to voters, and voters accept their responsibility, which is to listen, to weigh, and then to make their own choice".

Now this is just the reverse of the point of view that I have heard from the lips of both scientists and administrators. Let us, they have said, keep quiet about animal experiments. People do not want to hear about them, and if we direct their attention to this side of scientific activity, it will merely give stamping-ground to the anti-vivisectionists. Or perhaps they will say, nobody bothers much about rats and mice and guinea pigs; but whatever you do, leave cats and dogs out of it; these animals to-day occupy the place in men's hearts that horses did a century ago, and we should let them lie sleeping. But is this playing fair with the voters?

We need not concern ourselves with the abolitionists who are unreservedly opposed to any sort of animal experimentation. It is possible to respect their feelings, but not their way of conducting controversy. Perhaps I may digress a little in order to show how careful one has to be in the matter of antivivisection propaganda. In 1948 a deputation of antivivisectionists met the Home Secretary, and cited a number of alleged cruelties. Referring to the work of Mellanby on experimental rickets ${ }^{2}$ in puppies they quoted "animals were confined absolutely in kennels, $3 \mathrm{ft}$. 6 in. by $2 \mathrm{ft}$. 6 in., for 13 weeks. . : . These animals were most seriously attacked by 